\title{
Conformational Analysis of cis-Octahydropentalene, Insights on Bioactive Conformers
}

\author{
Mohammad Hossein Sakhaee ${ }^{\mathrm{a}}$, Sahar Sakhaee ${ }^{\mathrm{b}}$, Ahmad Takallouc $^{\mathrm{c}}$ and Nader Sakhaee ${ }^{\mathrm{d}, \mathrm{e} *}$ \\ ${ }^{a}$ Mashhad University of Medical Sciences, Mashhad, Iran \\ ${ }^{b}$ Islamic Azad University, Mashhad Branch, Mashhad, Iran.
}

'Department of Chemistry, Kharazmi University, Tehran, Iran

${ }^{d}$ Department of mathematics and Natural Sciences, Harris-Stowe State University, St. Louis, MO, 63108, USA

${ }^{e}$ Department of Chemistry, Southern Illinois University Edwardsville, Edwardsville, IL 62025, USA

*Corresponding author: Nader Sakhaee, Department of mathematics and Natural Sciences, Harris-Stowe State University, St. Louis, MO, 63108, USA; Department of Chemistry, Southern Illinois University Edwardsville, Edwardsville, IL 62025, USA

\begin{tabular}{l} 
ARTICLE INFO \\
\hline Received: 㓞 April 18, 2019 \\
Published: 㓞 April 23, 2019 \\
\hline Citation: Mohammad Hossein \\
Sakhaee, Sahar S, Ahmad T, Nader S. \\
Conformational Analysis of cis-Oc- \\
tahydropentalene, Insights on Bioac- \\
tive Conformers. Biomed J Sci \& Tech \\
Res 17(2)-2019. \\
BJSTR. MS.ID.002982.
\end{tabular}

ABSTRACT

The fluxional nature or so-called pseudorotation of cyclopentane has well been documented. Conformational dynamics in this molecule is so fast, that's even hard to populate one conformational form, in temperatures as low as $-100 \mathrm{oC}$ or $263 \mathrm{~K}$. cisOctahydropentalene contains two such fluxional molecule fused together. The molecule is not as rigid as its trans isomer, rather it's mobile and fluxional at both ends and rigid at the fused carbons. While rigid trans-decalin is known to occur in lipid bilayer to exert rigidity where its desired, cis-Octahydropentalene occurs in some natural products. Partial mobility of cis-Octahydropentalene can have profound effect on the function and biomedical properties of these natural products.

Keywords: cis-Octahydropentalene; Conformational Dynamics; Pseudorotation; Biomedical Properties; Partial Mobility; Conformational Analysis.

\section{Introduction}

The first complete pseudorotational model for conformational analysis of cyclopentane was introduced by Lipnick et al. [1] They Provided a wheel model of a cast of 20 conformers, arranged in a conformational wheel [2,3] to describe cyclopentane conformational flippings. Further attempts [4-12] to separate these conformers based on either energy or vibrational patterns mostly proved unsuccessful. Some 37 years later Laane et al. [7], provided a precise energy for twist and bent conformers $[13,14]$ showing how close the two conformers are in energy and vibrations. The concept of a spherical conformational landscape was introduced by our group back in 2016, to cyclopentane and some of its derivatives which helped fully explain their fluxional nature, confirmed by $1100-1600 \mathrm{~cm}^{-1}$ region of their Raman spectra. A first-attempt spherical conformational landscape was also presented for cis-octahydropentalene. In this paper we try to analyze the conformational preferences of Cis-Octahydropentalene in some natural products containing this fascinating molecule. Whether the mobility of this fused five membered ring system is of crucial importance to bioactivity of theses natural products is yet to be determined.

\section{Methods}

To study conformational Dynamics in cyclic molecules, most local density functional methods, slightly over estimate the barriers mostly due to their inability to account for van der Waals attractions in twist conformations $[15,16]$ While some density functional methods, in particular xb97xd, yield results that are both comparable and similar to those obtained by correlational methods like MP2. The $\omega b 97 x d$ (used here) can reliably result in accurate van der Waals considerations. The geometries computations were 
done at $\omega b 97 x d / 6-311+G(d)$ level [17-19], using Gaussian g09 package [18]. Larger basis sets like $6-311+G(d, p)$ proved no better accuracy. In fact, the cc-pVTZ basis sets were slightly better, some basic conformers and those with methyls were in some cases studied with cc-pVTZ basis set [19].

\section{Results and Discussion}

What happens if one move on to fused five membered ring systems, like Octahydropentalenes. Whether the same rationale can be used for these systems is to be discussed hereafter. Octahydropentalenes are five membered fused rings that can exist as cis and trans forms. The cis form is about $8 \mathrm{kcal} / \mathrm{mol}$ more stable than trans form and the reason for this stability could be well traced back in the skeletal conformation of 8 membered rings. Without the fusion in its carbonic skeleton, cis-Octahydropentalene resembles a very stable conformer of 8 membered rings. While trans Octahydropentalene without the fusion in the carbonic skeleton would resemble a high energy conformer of 8 membered rings, that will rapidly convert to more stable forms. The energy difference between these forms is about $9 \mathrm{kca} / \mathrm{mol}$. So regardless of how would the fusion contribute to the stability of cis/trans
Octahydropentalenes the trans-annular strain alone, will explain why the trans form is not as stable as cis.

Cis-Octahydropentalene is not only the most stable form but is rather a mobile molecule. There are 13 double envelope forms, 8 envelope half-chair forms and 2 double half-chair forms as well. In order to categorize and recognize better we'll look all the cis conformers in a direction that Hydrogens are in the back of the plane shown. Out of plane atom in the envelope forms are shown with a sign if coming toward us and with if protruding away. Out of plane carbons for half-chairs are shown the same way but not circled and in plane carbons are designated with a bold line. Energies and representative drawings are included here Figure 1 , Table 1 . This will help one classify and recognize all possible conformers of Octahydropentalene from simple 2-D drawings. Double envelope forms are the most stable conformers and among them there are four interesting $\mathrm{C}_{2}$ symmetric cousins of cis-decalin. Half-chair conformers lie between 4.5 to $6 \mathrm{kcal} / \mathrm{mol}$ higher than the double envelopes while cHCHC1 is $2.5 \mathrm{kcal} / \mathrm{mol}$ higher, and the other double half-chair is about $6.8 \mathrm{kcal} / \mathrm{mol}$ higher than the reference cEE3 conformer.
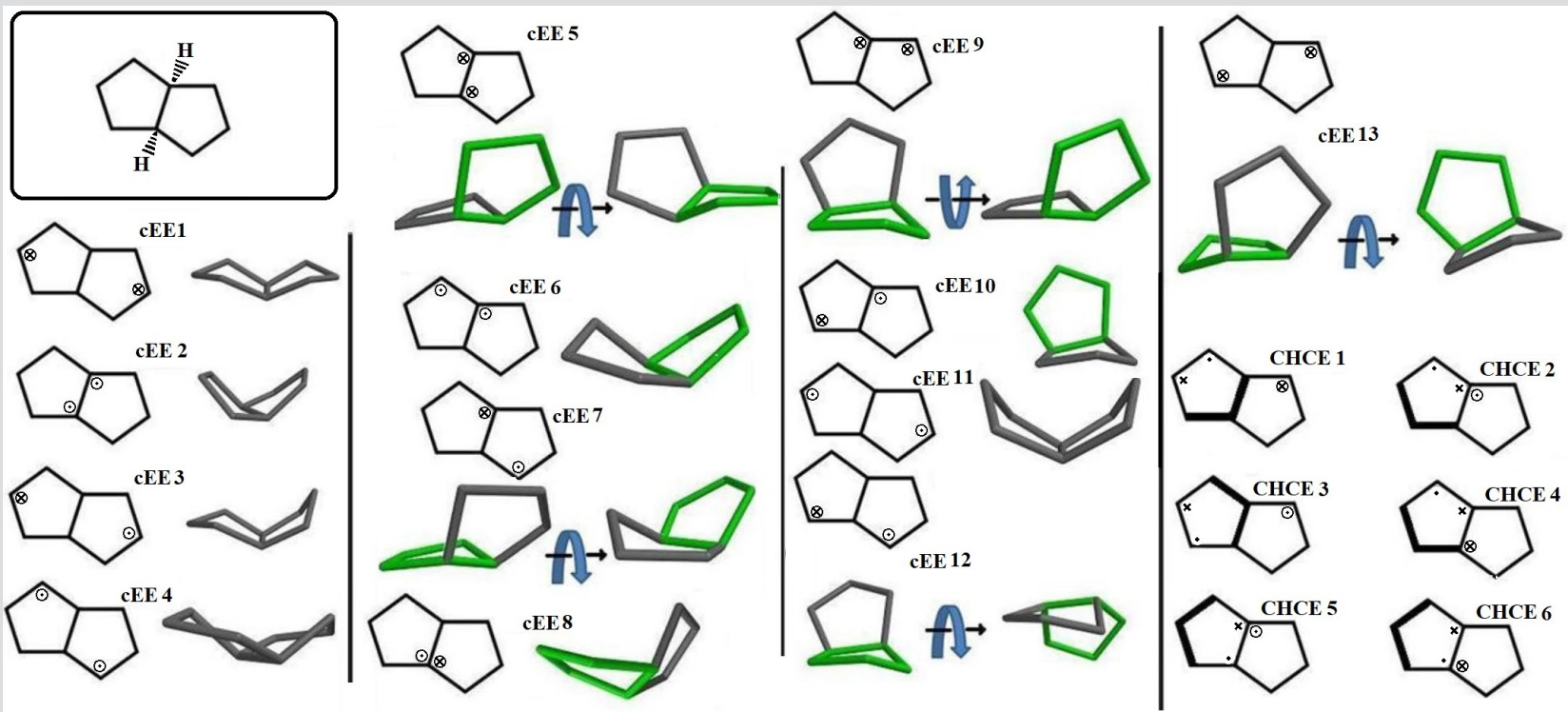

Figure 1: Cis-Octahydropentalene conformers (dots and encircled dots means coming toward us, Crosses and encircled crosses mean going away from us. For simplicity and systematic investigation, we'll look to the compound always with cis hydrogens going away from us)

Table 1: Conformational forms of Cis-Octahydropentalene (Energies and Symmetry).

\begin{tabular}{|c|c|c|c|}
\hline cis-Octahydropentalene & & DFT $\mathbf{\omega b 9 7 x d} / \mathbf{6 - 3 1 1 + G ^ { * }}$ & Erel(KCal/mol) \\
\hline Classes & Conformers & Point Groups & 1.2 \\
\cline { 2 - 4 } & cEE1 & C2v & 3.9 \\
\cline { 2 - 4 } & cEE2 & C2 & 0 \\
\cline { 2 - 4 } Double Envelopes & cEE3 & C2h & 2.1 \\
\cline { 2 - 4 } & cEE4 & C2 & 3.7 \\
\cline { 2 - 4 } & cEE5 & C2 & 4.2 \\
\cline { 2 - 4 } & cEE6 & C1 & 3.8 \\
\cline { 2 - 4 } & cEE7 & C1 & 3.9 \\
\cline { 2 - 4 } & cEE8 & C1 & 4.3 \\
\hline
\end{tabular}




\begin{tabular}{|c|c|c|c|}
\hline & cEE10 & $\mathrm{C} 1$ & 4.2 \\
\hline & cEE11 & $\mathrm{C} 2 \mathrm{v}$ & 1.7 \\
\hline & cEE12 & $\mathrm{C} 1$ & 2.4 \\
\hline & cEE13 & $\mathrm{C} 2$ & 2 \\
\hline \multirow{2}{*}{ Double Half Chair } & cHCHC1 & $\mathrm{C} 2$ & 2.5 \\
\hline & cHCHC2 & $\mathrm{C} 1$ & 6.8 \\
\hline \multirow{7}{*}{ Envelope -Half Chair } & cHCE1 & $\mathrm{C} 1$ & 5.5 \\
\hline & cHCE2 & $\mathrm{C} 1$ & 6.6 \\
\hline & cHCE3 & $\mathrm{C} 1$ & 5.6 \\
\hline & cHCE4 & $\mathrm{C} 1$ & 8.3 \\
\hline & cHCE6 & $\mathrm{C} 1$ & 8.3 \\
\hline & cHCE7 & $\mathrm{C} 1$ & 15.7 \\
\hline & cHCE8 & C1 & 12.1 \\
\hline
\end{tabular}

The big picture might be a little messy, but there are two distinct groups of conformers that show a full cycle of pseudo rotation. Clearly cEE1, 12, 12*, 7, 7* and cEE3, 9, 9*, 13, 13* can have a rapid full cycle conversion in the two separate platform $\mathrm{E}_{\mathrm{a}}$ and $\mathrm{E}_{\mathrm{b}}$ respectively. Conformers can switch between these two platforms via cHCE5 from cEE13, 13* to cEE12, 12*. The main role is played by conformer cEE1 and cEE3 in platforms $\mathrm{E}_{\mathrm{a}}$ and $\mathrm{E}_{\mathrm{b}}$ respectively. The cEE2 and cEE5 are the only isolated double envelopes, which are accessible only through half-chair forms and they share a similar structural feature. They both have their out of plane carbons as the junction carbons, and this serve as a lock to any flipping by the mechanism explained so far. Cis-Octahydropentalenes not only more stable than their trans isomers but also are of more frequent occurrences in nature. Cedrol, Meldrum's acid, Aburtubolactam, $(-)$ - -Cederene and (+)- -Cederene to mention a few have all a cis octahydropentalene skeletal substructure. Natural cedrol and Cederenes are the essential components in cedar oils in most coniferous trees.

They are used as fragrance in sprays, perfumes and also as antibacterial agents and fungicides. Aburtubolactam is a novel inhibitor of superoxide anions isolated from a marine organism. The above mentioned Cedrols and Cedrernes are all metabolized from a carbocation intermediate with a major conformational Preference for $\mathrm{CHCHC1}$, and upon conversion to terpenoid alcohols and olefins assume cEE4 conformational form in equilibrium with a minor conformer, cEE3. The cEE3 Conformer looks quiet magical cause it's a major conformer and might be the one with higher biomedical activity. Figure 2 summarizes all the above-mentioned conformational preferences seen in these natural products.

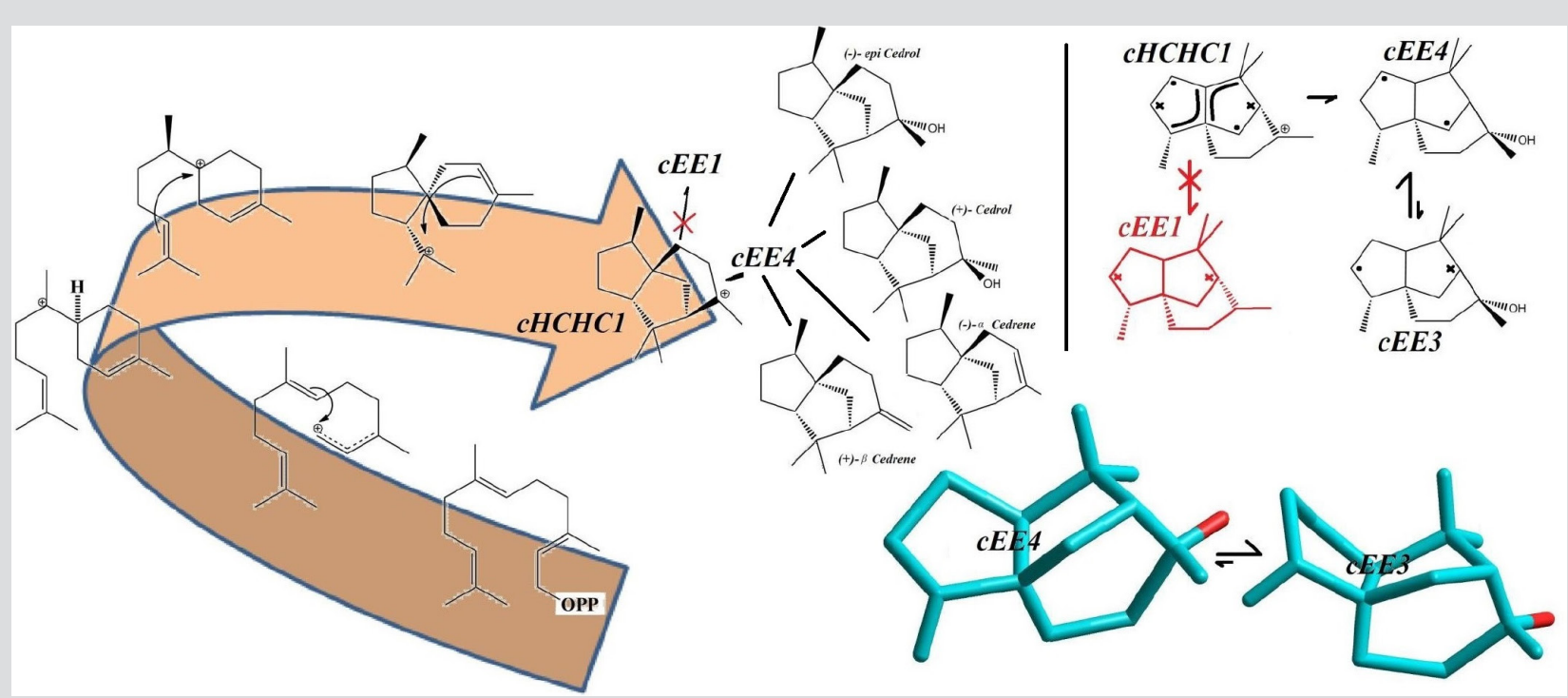

Figure 2:Conformational Preferences in olefinic and oxygenated sesquiterpenes, Cedrenes and Cedrols (Convention used to show the stereochemistry of the rings is the same used in Figure 1).

\section{Conclusion}

In summery it's been shown that a full conformational analysis of cyclopantane helps explain the intricate mechanism of interconversion between Envelope and half chair conformers. The same rationale were then extended to the case of fused five membered rings , Octahydropentalenes, were a newly proposed convention of their 2-D drawings help one classify all possible conformers. Here the nature of fusion imposed restrictions on mobility of the fused ring and some conformers are of higher energy content and thus not readily accessible. While the fusion imposed severe restriction on trans-octahydropentalene it cannot oppose the fluxionl nature 
in cis-Octahydropentalene, which leaves molecule rather mobile. Conformational studies are yet to be done in much more details for medium sized and larger ring molecules. Conformational analysis on big Macrolide are making it possible to track dominant conformational form and their role on bioactivity. Such an analysis would be the most valuable tool ever, to fuel the drug design and biomedical research.

\section{References}

1. Lipnick RL (1974) NMR spectroscopy of cyclopentane derivatives. III. Methylcyclopentane. Journal of the American Chemical Society 96(9): 2941-2948.

2. Hoffmann RW (2000) Conformation design of open-chain compounds. Angewandte Chemie International Edition 39(12): 2054-2070.

3. Saran A, Perahia D, Pullman B (1973) Molecular orbital calculations on the conformation of nucleic acids and their constituents. Theoretica chimica acta $30(1)$ : 31-44.

4. Pitzer KS, Donath WE (1993) Conformations and strain energy of cyclopentane and its derivatives, Molecular Structure and Statistical Thermodynamics: Selected Papers of Kenneth S Pitzer. World Scientific p. 98-103.

5. Kilpatrick JE, Pitzer KS, Spitzer R (1947) The thermodynamics and molecular structure of cyclopentane1, Journal of the American Chemical Society 69(10): 2483-2488.

6. Allinger NL, Hirsch JA, Miller MA, Tyminski IJ, Van Catledge FA (1968) Conformational analysis. LX. Improved calculations of the structures and energies of hydrocarbons by the Westheimer method. Journal of the American Chemical Society 90(5): 1199-1210.

7. Laane J (1969) Far-Infrared Spectrum and the Barrier to Pseudorotation of Silacyclopentane. The Journal of Chemical Physics 50(5): 1946-1951.

8. Wertz D (1969) Far-Infrared Absorption Spectrum and Pseudorotation of the Thiacyclopentane Molecule. The Journal of Chemical Physics 51(5): 2133-2136.

ISSN: 2574-1241

DOI: 10.26717/BJSTR.2019.17.002982

Nader Sakhaee. Biomed J Sci \& Tech Res

(c) This work is licensed under Creative

Submission Link: https://biomedres.us/submit-manuscript.php
9. Green W, Harvey A, Greenhouse J (1971) Spectroscopic Determination of the Pseudorotation Barrier in Selenacyclopentane. The Journal of Chemical Physics 54(3): 850-856.

10. Carreira L, Lord R (1969) Far-Infrared Spectra of Ring Compounds. V. Ring-Puckering Potential Functions of Some Oxygen-Containing Molecules. The Journal of Chemical Physics 51(8).

11. During J, Willis J (1970) Spectra and Structure of Small Ring Compounds. XIX. Vibrational Analysis and the Barrier to Pseudorotation of Germylcyclopentane. The Journal of Chemical Physics 52(12): 61086119.

12. Bauman L, Laane J (1988) Pseudorotation of cyclopentane and its deuterated derivatives. The Journal of Physical Chemistry 92(5): 10401051.

13. Brügger G, Frey HM, Steinegger P, Balmer F, Leutwyler S (2011) Accurate determination of the structure of cyclohexane by femtosecond rotational coherence spectroscopy and ab initio calculations. The Journal of Physical Chemistry A 115(34): 9567-9578.

14. Kowalewski P, Frey HM, Infanger D, Leutwyler S (2015) Probing the structure, pseudorotation, and radial vibrations of cyclopentane by femtosecond rotational Raman coherence spectroscopy. The Journal of Physical Chemistry A 119(45): 11215-11225.

15. Halgren TA (1996) Merck molecular force field. II. MMFF94 van der Waals and electrostatic parameters for intermolecular interactions. Journal of Computational Chemistry 17: 520-552.

16. Giesen DJ, Gu MZ, Cramer CJ, Truhlar DG (1996) A universal organic solvation model. The Journal of organic chemistry 61(25): 8720-8721.

17. Shao Y, Molnar LF, Jung Y, Kussmann J, Ochsenfeld C, et al. (2006) Advances in methods and algorithms in a modern quantum chemistry program package. Physical Chemistry Chemical Physics 8: 3172-3191.

18. Frisch M, Trucks G, Schlegel HB, Scuseria GE, Robb MA, et al. (2009) Gaussian 09, revision D. 01, Gaussian, Inc., Wallingford CT, England.

19. Hratchian HP, Parandekar PV, Raghavachari K, Frisch MJ, Vreven T (2008) QM: QM electronic embedding using Mulliken atomic charges: Energies and analytic gradients in an ONIOM framework. The Journal of chemical physics 128(3): 034107.

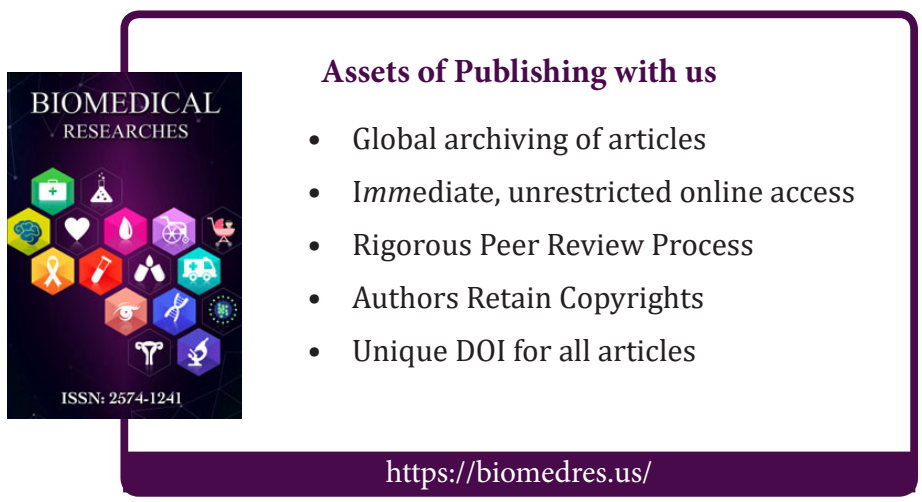

